\title{
PLASMA-OPTICAL EFFECT IN GaAs PIN PHOTODIODES
}

\author{
M. A. GRADO CAFFARO \\ Polytechnic University of Madrid, Spain \\ M. GRADO CAFFARO \\ Telecommunication Engineer (Senior), Scientific Research Council, Madrid, Spain \\ (Received April 25, 1992; in final form June 11, 1992)
}

This paper is devoted to the analysis of the Plasma-Optical Effect in GaAs PIN photodiodes operating at the infrared range. An approximated expression for the variation of the refractive index in the intrinsic zone of a AsGa PIN photodiode is obtained. This variation is induced by the charge of the device. The approach developed by us is in a good agreement with experimental works. Moreover, an application concerning high-frequency ICs is outlined.

\section{INTRODUCTION}

It is well known that the Plasma-Optical effect is based on the following fact: in a semiconductor the electrons of the conduction band and the holes of the valence band behave as very free carriers. The above assumption is simple but, in principle, it constitutes a valid approach for our purpose. At optical frequencies, the variation of the refractive index due to the charge within the device is small. In this work, our device is a GaAs PIN photodiode and the above variation is less small when the device detects optical power belonging to the infrared range (in particular, to the far infrared range). By means of an adequate Taylor expansion, an accurate formula for the modified index of refraction is calculated.

\section{CALCULATIONS}

It is well-known that the local refractive index of a semiconductor under the free carrier approach is given by the following expression:[1]

$\eta=\eta_{\infty}\left[1-\frac{e^{2}}{\varepsilon \omega^{2}}\left(\frac{n}{m_{n}^{*}}+\frac{p}{m_{p}^{*}}\right)\right]^{1 / 2}$

where $\eta$ is the refractive index at an angular frequency $\omega, \eta_{\infty}$ is the refractive index at very high frequencies $(\omega \rightarrow \infty), e$ is the electronic charge, $\varepsilon$ is the dielectric permittivity, $n$ and $p$ the electron and hole concentrations, respectively, and $m_{n}^{*}$ and $m_{p}^{*}$ the electron and hole effective masses, respectively. Equation (1) is 
established by assuming that free-carrier effective mass does not depend on carrier concentration. First, we will consider this crude approach and later we will develop a more accurate scheme.

Assuming a PIN photodiode based on isotropic GaAs, for the intrinsic region of the diode we have $p=n=n_{i}$ in thermal equilibrium and by considering (1) we can write:

$\eta=\eta_{\infty}\left(1-\frac{e^{2} n_{i}}{\varepsilon \omega^{2} m^{*}}\right)^{1 / 2}$

where $n_{i}$ is the intrinsic carrier concentration and $m^{*}$ is the reduced effective mass namely, $m^{*}=m_{n}^{*} m_{p}^{*} /\left(m_{n}^{*}+m_{p}^{*}\right)$. For the far infrared range, a second order Taylor's expansion in (2) will be considered. We have $\left(1 / \omega^{2}\right)=x$ and:

$(1-a x)^{1 / 2} \cong 1-\frac{a x}{2}-\frac{a^{2} x^{2}}{8}$

with: $a=\frac{e^{2} n_{i}}{\varepsilon m^{*}}$. From (2) and (3) we get:

$\eta \cong \eta_{\infty}\left[1-\frac{e^{2} n_{i}}{2 \varepsilon m^{*} \omega^{2}}\left(1+\frac{a}{4 \omega^{2}}\right)\right]$

From (4) it is deduced:

$\Delta \eta=\eta_{\infty}-\eta=\frac{\eta_{\infty} \omega_{0}^{2}}{2 \omega^{2}}\left(1+\frac{\omega_{0}^{2}}{4 \omega^{2}}\right)$

where $\omega_{0}=e\left(n_{i} / \varepsilon m^{*}\right)^{1 / 2} \equiv a^{1 / 2}$ is the so-called plasma resonant angular frequency. Expression (5) shows the refractive index perturbation in the intrinsic zone of our photodiode (Fig. 1).

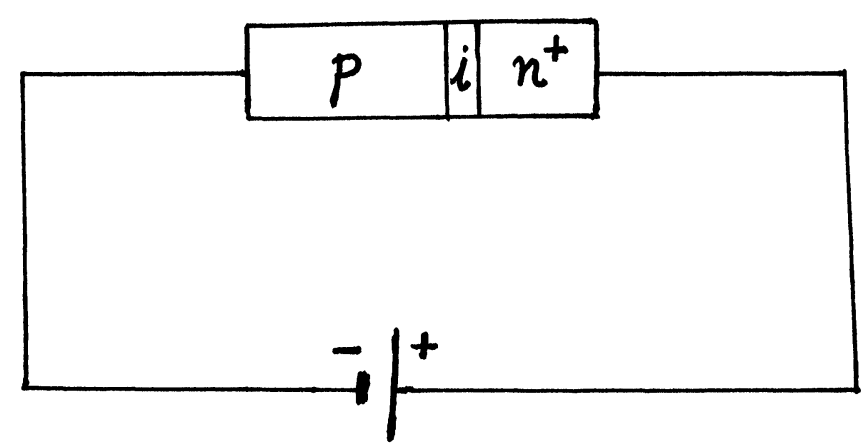

FIGURE 1 PIN photodiode with strongly doped $n$-zone. 
Now, we will develop a more realistic approach to calculate the variation of the refractive index for the three regions of the photodiode. Soref and Bennett[2] have developed a theoretical model establishing the following equation:

$\Delta \eta=\frac{\eta_{\infty} e^{2}}{2 \varepsilon \omega^{2}}\left(\frac{\alpha_{n} n^{4 / 5}}{m_{n}^{*}}+\frac{\alpha_{p} p^{21 / 20}}{m_{p}^{*}}\right)$

where $\alpha_{n}$ and $\alpha_{p}$ are corrective factors corresponding to electrons and holes, respectively. Also, Koskowich et al. have developed a model finding similar results[3].

Eq. (6) is adequate for silicon. However, we have obtained a more suitable formula for GaAs, namely:

$\Delta \eta=\frac{\eta_{x} e^{2}}{2 \varepsilon \omega^{2}}\left(\frac{\alpha_{n} n}{m_{n}^{*}}+\frac{\alpha_{p} p}{m_{p}^{*}}\right)$

Eq. (7) is in a good agreement with experimental works of various researchers[4]. Eqs. (6) and (7) have been obtained under the assumption by which free-carrier effective mass depends on carrier concentration. By using Equation (1) under this assumption, we find

$\eta=\eta_{x}\left[1-\frac{e^{2}}{\varepsilon \omega^{2}}\left(\frac{\alpha_{n} n}{m_{n}^{*}}+\frac{\alpha_{p} p}{m_{p}^{*}}\right)\right]^{1 / 2}$

By using a Taylor series in terms of $1 / \omega^{2}$ for expression (8), it is derived:

$\Delta \eta=\frac{\eta_{\infty} e^{2}}{2 \varepsilon \omega^{2}}\left(\frac{\alpha_{n} n}{m_{n}^{*}}+\frac{\alpha_{p} p}{m_{p}^{*}}\right)\left(1+\frac{\omega_{0}^{2}}{4 \omega^{2}}\right)$

with:

$\omega_{0}=e\left[\frac{1}{\varepsilon}\left(\frac{\alpha_{n} n}{m_{n}^{*}}+\frac{\alpha_{p} p}{m_{p}^{*}}\right)\right]^{1 / 2}$

hence Eq. (9) reduces to:

$\Delta \eta=\frac{\eta_{\infty} \omega_{0}^{2}}{2 \omega^{2}}\left(1+\frac{\omega_{0}^{2}}{4 \omega^{2}}\right)$

For the intrinsic region of the PIN photodiode we have $p=n=n_{i}$ and Eq. (9) reduces to Eq. (11) with:

$\omega_{0}^{2}=\frac{e^{2} n_{i}}{\varepsilon}\left(\frac{\alpha_{n}}{m_{n}^{*}}+\frac{\alpha_{p}}{m_{p}^{*}}\right)$ 
On the other hand, for the $p$ zone, we have $p \gg n, m_{p}^{*}>m_{n}^{*}$ and Eq. (9) transforms in Eq. (11) where

$\omega_{0}^{2} \cong \frac{e^{2} \alpha_{p} p}{\varepsilon m_{p}^{*}}$

since employing quantum arguments we have obtained $\alpha_{p}>\alpha_{n}$ (for GaAs).

Finally, for the $n$ zone we have $n \gg p, m_{p}^{*}>m_{n}^{*}$ and Eq. (9) reduces to Eq. (11) with:

$\omega_{0}^{2} \cong \frac{e^{2} \alpha_{n} n}{\varepsilon m_{n}^{*}}$

\section{RESULTS AND DISCUSSION}

In our context (PIN photodiodes) Equation (12) represents the crucial result since this expression corresponds to the intrinsic region of the PIN photodiodes and it is well-known that the lightwaves fall over that region. Equation (12) refers to the far infrared range and is in a good agreement with experimental works[5].

On the other hand, the variation of the refractive index of an intrinsic semiconductor due to the charge in this semiconductor, constitutes an important element in the context of the charge-sensing optical probing systems [6,7]. Basically, a plasma-optical probing system detects the refractive index variation by interferometrically sensing the differential phase shift between two optical beams; these beams are a probe beam, which passes through a device, and a reference beam, which passes through a near zone without a device. In our case the device related to the probe beam is a GaAs PIN photodiode.

The charge-sensing optical probing technique is a relevant tool for GaAs integrated circuits in order to detect picosecond electric signals. Charge-sensing optical probing systems have high sensitivity and they are non-invasive.

\section{REFERENCES}

1. F. Wooten: "Optical Properties of Solids". Academic Press, New York, 1972 (pp. 52-55).

2. R. A. Soref, B. R. Bennett: IEEE J. Quantum Electron. $Q E-23,123$ (1987).

3. G. N. Koskowich, R. B. Darling, M. Soma: Phys. Rev. B 38, 1281 (1988).

4. H. K. Heinrich, D. M. Bloom, B. R. Hemenway: Appl. Phys. Lett. 48, 1066 (1986).

5. B. R. Hemenway, H. K. Heinrich, J. H. Goll, Z. Xu, D. M. Bloom: Electron Device Lett. EDL-8, 344 (1987).

6. U. Keller, S. K. Diamond, B. A. Auld, D. M. Bloom. Appl Phys. Lett. 53, 388 (1987).

7. H. K. Heinrich. IBM J. Res. and Development, 34, No. 2/3, 164 (1990). 

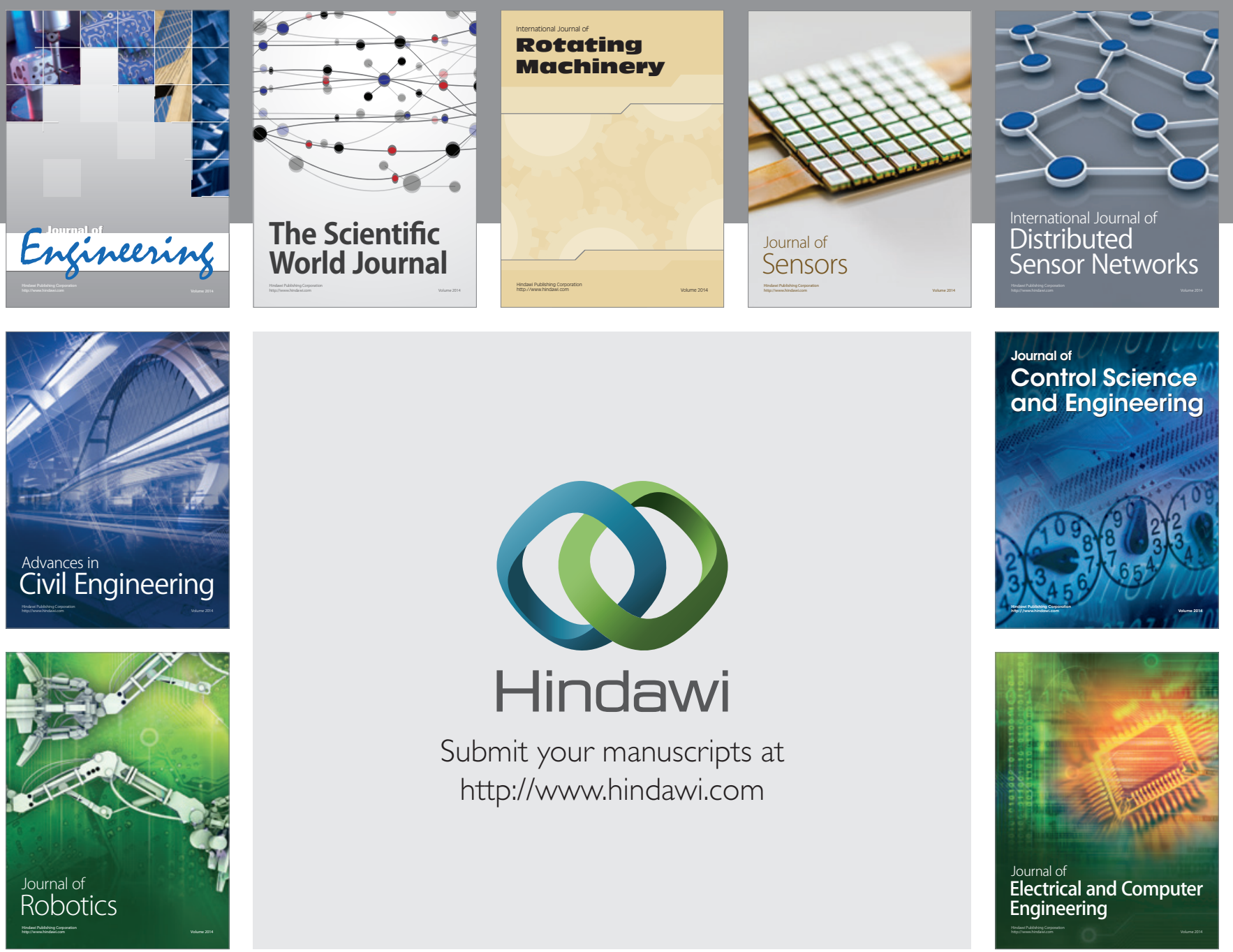

Submit your manuscripts at

http://www.hindawi.com
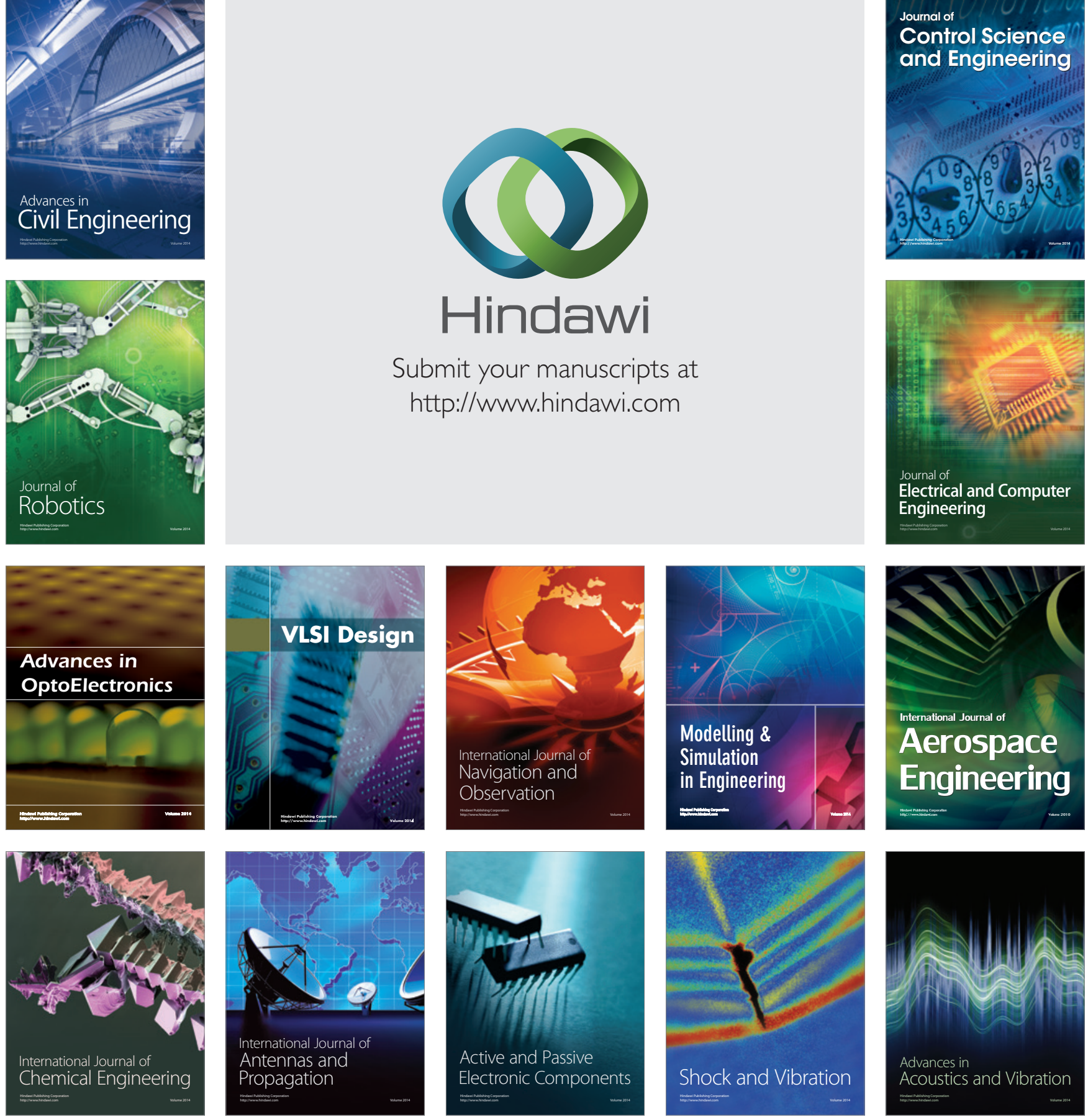